\title{
Weak ionization of the global ionosphere in solar cycle 24
}

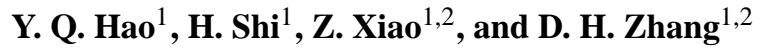 \\ ${ }^{1}$ Department of Geophysics, Peking University, Beijing, China \\ ${ }^{2}$ State Key Laboratory of Space Weather, Center for Space Science and Applied Research, Chinese Academy of Sciences, \\ Beijing, China
}

Correspondence to: Y. Q. Hao (hao@pku.edu.cn)

Received: 8 December 2013 - Revised: 7 June 2014 - Accepted: 10 June 2014 - Published: 21 July 2014

\begin{abstract}
Following prolonged and extremely quiet solar activity from 2008 to 2009 , the 24th solar cycle started slowly. It has been almost 5 years since then. The measurement of ionospheric critical frequency $(f o \mathrm{~F} 2)$ shows the fact that solar activity has been significantly lower in the first half of cycle 24 , compared to the average levels of cycles 19 to 23 ; the data of global average total electron content ( $\overline{\mathrm{TEC}})$ confirm that the global ionosphere around the cycle 24 peak is much more weakly ionized, in contrast to cycle 23 . The weak ionization has been more notable since the year 2012, when both the ionosphere and solar activity were expected to be approaching their maximum level. The undersupply of solar extreme ultraviolet (EUV) irradiance somewhat continues after the 2008-2009 minimum, and is considered to be the main cause of the weak ionization. It further implies that the thermosphere and ionosphere in the first solar cycle of this millennium would probably differ from what we have learned from the previous cycles of the space age.
\end{abstract}

Keywords. Ionosphere (ionization mechanisms, solar radiation and cosmic ray effects) - radio science (ionospheric physics)

\section{Introduction}

According to the observation of solar sunspots, the previous solar cycle hit its minimum in late 2008 and early 2009; then solar cycle 24 (SC24) started (SIDC-team, 1954-2013; Owens et al., 2011). The solar minima of cycles 23-24 covered the years 2008-2009, which was an extremely quiet period with a solar sunspot number (SSN) close to 0 and in general deeper and longer than other recent minima (Russell et al., 2010). Satellite measurements showed unusually low solar extreme ultraviolet (EUV) irradiance during the recent minimum (Solomon et al., 2010; Lockwood, 2011), and the difference between 2008 and the previous minimum year 1996 was estimated to be probably close to $10 \%$ and within limits of $6-15 \%$ by various authors (see Laštovička, 2013, and the references therein).

The input of solar energy heats the thermosphere and ionizes the neutral atmosphere to produce the ionosphere. Along with the unprecedentedly low solar EUV irradiance in 2008 2009 , both the thermospheric density and the ionospheric electron density dropped to record lows. Numerous studies have reported the unusual behavior of the thermosphereionosphere system during this solar minimum. The density of the thermosphere from 2007 to 2009, for instance, was found to be lower than at any time since the beginning of the space age, according to the analyses of the historical archive of atmospheric drag on satellite orbits (Emmert et al., 2010). In particular, the thermospheric density at $400 \mathrm{~km}$ was lower by $30 \%$ in 2009 in comparison to the solar cycle $22 / 23 \mathrm{~min}$ imum in 1996 (Solomon et al., 2010; Emmert et al., 2010). Quantitative analysis indicates that solar EUV irradiance, as the primary contributor to the ionization, created a record low ionospheric electron density during the cycle $23 / 24 \mathrm{~min}$ imum. Both the space-borne measurements of EUV irradiance and the proxy $10.7 \mathrm{~cm}$ solar radio flux (F10.7 index) in this period were found to be lower than other recent minima, and accordingly the ionospheric critical frequency $f_{o} \mathrm{~F} 2$ and total electron content (TEC) shrank to a record low level (Liu et al., 2011a; Araujo-Pradere et al., 2011).

Following the SC23-24 minimum, the new cycle began with some uncommon characteristics as well. The recent three solar minima occurred in 1976, 1986 and 1996, respectively. It would have been expected that SC24 should come shortly after the year 2006 minimum, but the prolonged 
minimum lasted till 2008, and the new cycle actually initiated in 2009 according to the sunspot record. Nevertheless, the Sun did not start displaying appreciable activity until 2010, and the rise in activity has been slow relative to most other cycles during the last century (Russell et al., 2010; Solanki and Krivova, 2011). Before the prolonged minimum, solar astronomers had made predictions of the amplitude and timing of the upcoming SC24. However, the results showed significant discrepancies. The peak SSN was expected to be in a wide range of 42-185, and the solar maximum was predicted to occur between the years 2009 and 2012 (Pesnell, 2008, 2012).

Some studies estimated the new cycle to be less active. For instance, according to the periodicities in solar activity, SC24 was expected to be considerably smaller than SC23, with peak SSN expected to be $42 \pm 34$ (Clilverd et al., 2006). Based on the dynamo model and observations of the solar polar magnetic field, SC24 was predicted to peak in 2011 and have a maximum smoothed monthly SSN of $75 \pm 8$ (Svalgaard et al., 2005). So far they are more likely to be the right ones, because the later experimental observations during the cycle 23/24 minima and after the initiation of SC24 have indicated a potentially weak cycle. Some estimations of the new cycle have therefore been updated to an ultra low level (e.g., Kane, 2010). The observed SSN of the 36th month (November 2011) of SC24 is 61.1 and obviously lower than the 90.5 of SC23 (May 1999), from which Kane (2013) predicted that SC24 will have a maximum amplitude of about 77.0 \pm 13.4. Drawn upon initial observations of sunspot number and latitude in SC24, Owens et al. (2011) reached a similar result, that the SSN is to peak around $65 \pm 10$ at the middle/end of 2012. Moreover, a consensus has been reached by NOAA's expert panel that the next solar cycle will be below average in intensity, with a peak SSN of 90, and solar maximum is expected to occur in May 2013 (http://www.swpc.noaa.gov/SolarCycle/SC24/index.html).

If the above predictions of peak time were true, SC24 would have reached or approached its maximum, up to the time of this writing, October 2013. Nearly 5 years have passed since the initiation of SC24. How has the ionosphere behaved in the first half cycle? Has the atmosphere been ionized as in normal solar maximum years, or has it consequently stayed low following the preceding unusual minimum and the slow ascent of SC24? To answer these questions, we interpreted the experimental data of ionospheric foF 2 and TEC, and compare the measurements in $\mathrm{SC} 24$ to those in other recent cycles. The ionospheric anomaly in the first half of SC24 is therefore outlined, and the role of an inactive Sun in producing weak ionization is discussed.

\section{Data and results}

Ionosonde and dual-frequency Global Navigation Satellite System (GNSS) receivers are two common kinds of

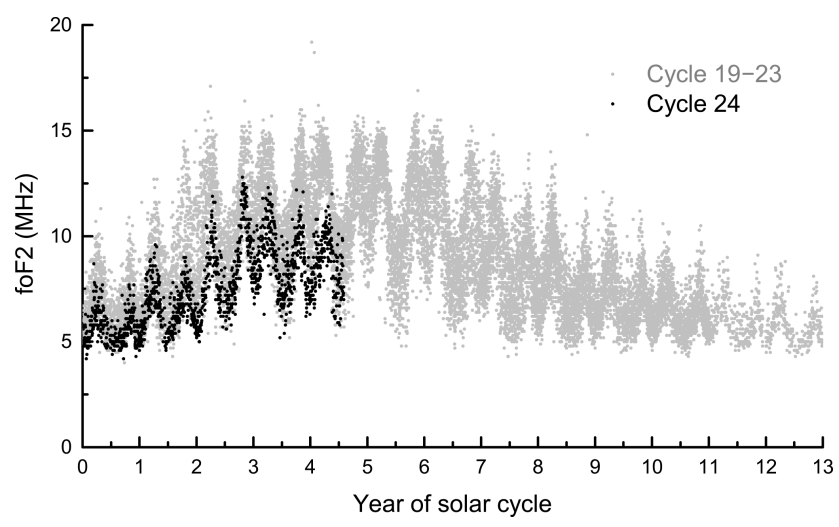

Figure 1. Ionospheric $f o \mathrm{~F} 2$ at 14:00 LT measured by the Kokubunji ionosonde since solar cycle 19. The data points for SC19-23 are in grey, and those for SC24 are in black. The number labels on the $x$ axis denote the year counted from the beginning of each solar cycle. As has been explained in Sect. 2.1, the nearby January is taken as the start time of a solar cycle, e.g., for SC24 $x=0$ corresponds to January 2009. This $x$ axis convention also applies to the other figures in this paper.

ground-based instrument that continuously measure parameters of the ionosphere. Ionosonde data of $f o \mathrm{~F} 2$ (in $\mathrm{MHz}$ ) reflect the peak electron density in the ionosphere, while the TEC data derived from GNSS receiver observations give the total ionospheric electron content that is integrated along altitude. Both the $f o F 2$ and TEC data have been used in numerous ionospheric studies, and due to the long-term continuity they are especially valuable for investigations over solar cycles. In this work, we collect $f o \mathrm{~F} 2$ data of the Kokubunji ionosonde covering the period from SC19 to date, and global TEC data since the year 1996 that allow us to compare SC24 and $\mathrm{SC} 23$.

\subsection{Single-station foF2 from solar cycles 19 to 24}

Kokubunji (geographic $35.7^{\circ} \mathrm{N}, 139.5^{\circ} \mathrm{E}$, code TO535) is one of the most historical ionosonde stations. The $f o \mathrm{~F} 2$ data at 14:00 LT from January 1954 to September 2013 are used in the analysis to show the differences between SC24 and the preceding five cycles since the space age began. The start time, end time and peak SSN of these cycles are listed in Table 1. In Fig. 1, the $f o \mathrm{~F} 2$ data over SC19 to SC24 are arranged with SC24 (black points) superimposed on the previous five cycles (grey points). At this time, the nearby January is taken as the start time of a solar cycle, e.g., SC24 began at January 2009. This is for the purpose of retaining the equinoctial peaks, considering that the ionospheric $f o \mathrm{~F} 2$ and TEC usually show semiannual variation with values peaking at equinoxes. From Fig. 1 we can tell that $\mathrm{SC} 24 f o \mathrm{~F} 2$ is obviously below the average level of previous cycles. Since the beginning of $\mathrm{SC} 24$, the $f o \mathrm{~F} 2$ value has been roughly along the bottom edge of the SC19-23 envelope. 
Table 1. The start time, peak time and peak sunspot number of SC19 to SC24.*

\begin{tabular}{cllr}
\hline Cycle & Start time & Peak time & Peak SSN \\
\hline 19 & Mar 1954 & Mar 1958 & 201.3 \\
20 & Oct 1964 & Dec 1968 & 110.1 \\
21 & Jun 1976 & Dec 1979 & 164.5 \\
22 & Sep 1986 & Jul 1989 & 158.5 \\
23 & May 1996 & Apr 2000 & 120.8 \\
24 & Feb 2009 & Feb 2012 & 66.9 \\
\hline
\end{tabular}

* The start time, end time and peak SSN of solar cycles are identified based on a 13-month smoothed monthly sunspot number record (SIDC-team, 1954-2013). The newest monthly SSN available at the time of this writing is from September 2013, and the latest 13-month smoothed SSN value is from March 2013. Accordingly, the peak SSN of SC24 till now is found to be 66.9 in February 2012.

To quantify the deviation of SC24 from the historical average, we calculated monthly mean $f o \mathrm{~F} 2$ values for $\mathrm{SC} 24$ and all the previous cycles. Along with the mean value of SC24, the monthly mean $f o \mathrm{~F} 2$ of SC19-23 and its standard deviation $( \pm 1 \sigma)$ are also displayed in Fig. 2. Since the very beginning of SC24 (year 2009), foF2 has been lower than the monthly means of SC19-23, and most of the time $f o \mathrm{~F} 2$ is actually at the level of $-1 \sigma$. After 2012, $f o \mathrm{~F} 2$ was even lower. In many months of the fourth and fifth years of SC24 (2012 and 2013), $f o \mathrm{~F} 2$ was well below the $-1 \sigma$ value. The percentage departure of monthly mean SC24 foF2 $\left(f_{24}\right)$ from the average level of SC19-23 $\left(f_{19-23}\right)$ and minus one standard deviation $\left(f_{19-23}-1 \sigma\right)$ is depicted in Fig. 3a and b, respectively. In 2009 and $2010, f_{24}$ was lower than $f_{19-23}$ by 10-20\%; after 2011 the difference was about $-20 \%$, and particularly in January of 2011 and 2013 (northern winter) the departure increased to $30-40 \%$. Figure $3 \mathrm{~b}$ confirms that $f_{24}$ is generally at the level of $\left(f_{19-23}-1 \sigma\right)$ before the year 2011 , and after that it falls outside of $-1 \sigma$ most of the time, especially in northern winters.

The $f o \mathrm{~F} 2$ measurements of the Kokubunji ionosonde has shown that the ionosphere of SC24 is significantly lower than historical records of SC19-23. During low solar activity years, the departure of $f_{o} \mathrm{~F} 2$ was $-20 \%$ or $-1 \mathrm{MHz}$ at most; in recent high-activity years, the departure could reach $-40 \%$ or $-5 \mathrm{MHz}$. This result represents the feature of the mid-latitude ionosphere in East Asia that is weakly produced in the first half of SC24, and this feature is in particular remarkable in association with unusual slow climbing of solar activity as SC24 proceeding.

\subsection{Global average TEC in solar cycles 23 and 24}

The worldwide network of GNSS receiver stations maintained by the International GNSS Service (IGS) is outstanding in providing global ionospheric sensing. Analysis centers routinely produce global ionospheric maps (GIMs) in IONosphere map EXchange (IONEX) format, with a two-hourly

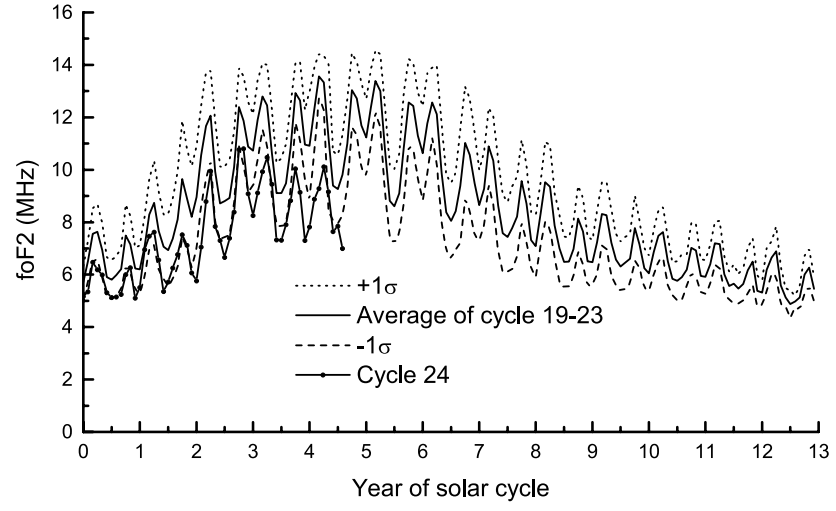

Figure 2. Similar to Fig. 1, but displayed are the monthly mean $f o \mathrm{~F} 2$ of both SC24 and SC19-23. The standard deviations $( \pm 1 \sigma)$ about the mean $f o \mathrm{~F} 2$ of SC19-23 are shown in dotted and dashed lines as well.

cadence and a spatial resolution of $5^{\circ}$ in longitude and $2.5^{\circ}$ in latitude (Schaer et al., 1998; Mannucci et al., 1998). In the present study we use the global TEC data provided by the Center for Orbit Determination in Europe (CODE) at the Astronomical Institute of University of Bern (AIUB) (http://www.aiub.unibe.ch/). The GIMs from January 1995 are available at CODE, with a temporal resolution of 1 day at first, and enhanced to $2 \mathrm{~h}$ after April 1998. Considering that SC23 started in 1996, this is an excellent database for comparing the ionosphere of the first half cycle in $\mathrm{SC} 24$ to that in $\mathrm{SC} 23$.

The CODE GIMs data on every day from January 1996 to September 2013 were averaged globally to calculate the daily global average TEC (TEC) in a similar way to Hocke (2008) and Liu et al. (2009). This index is thought to be able to reduce local noise, represent the global nature of the ionosphere, and excellently track the solar cycle variation and solar rotation modulations; thus it can be used as a practical ionospheric index (Hocke, 2008; Liu et al., 2009).

The calculated $\overline{\text { TEC }}$ since 1996 are shown in Fig. 4 to compare $\mathrm{SC} 24$ to $\mathrm{SC} 23$, with the start and end times of $\mathrm{SC} 23$ taken to be the same as in the $f o \mathrm{~F} 2$ analysis (January 1996 and December 2008, respectively). Unlike the behavior of $f o \mathrm{~F} 2$, the $\overline{\mathrm{TEC}}$ values in $\mathrm{SC} 24$ were not lower than $\mathrm{SC} 23$, at least in the first three years (2009-2011). Sometimes the values were even higher than SC23, i.e., at the equinoxes of years 2010 and 2011. The enhancement was particularly remarkable around the fall equinox of 2011, as the Sun became more and more active. However, the trend was reversed in 2012 and the $\overline{\text { TEC }}$ values began to be lower than SC23. This feature was further strengthened, with the value peaking at $30 \mathrm{TECu}$ in the first half year of 2013, and by contrast the peak value in SC23 reached almost $60 \mathrm{TECu}$ correspondingly.

Similar to the analysis of $f_{o} \mathrm{~F} 2$, the monthly mean value and standard deviation of TEC were calculated and depicted 


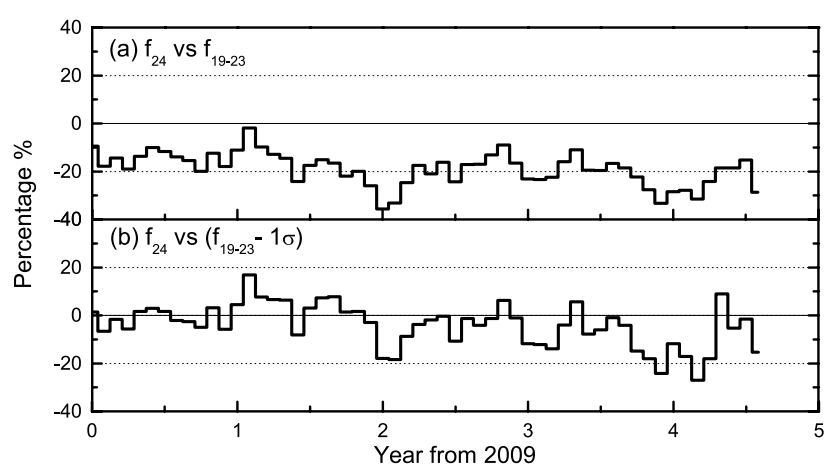

Figure 3. The percentage deviation of $\mathrm{SC} 24 f o \mathrm{~F} 2\left(f_{24}\right)$ from recent solar cycles, (a) compared to the mean value of SC19-23 ( $\left.f_{19-23}\right)$ and (b) compared to minus one standard deviation about the SC1923 mean value $\left(f_{19-23}-1 \sigma\right)$.

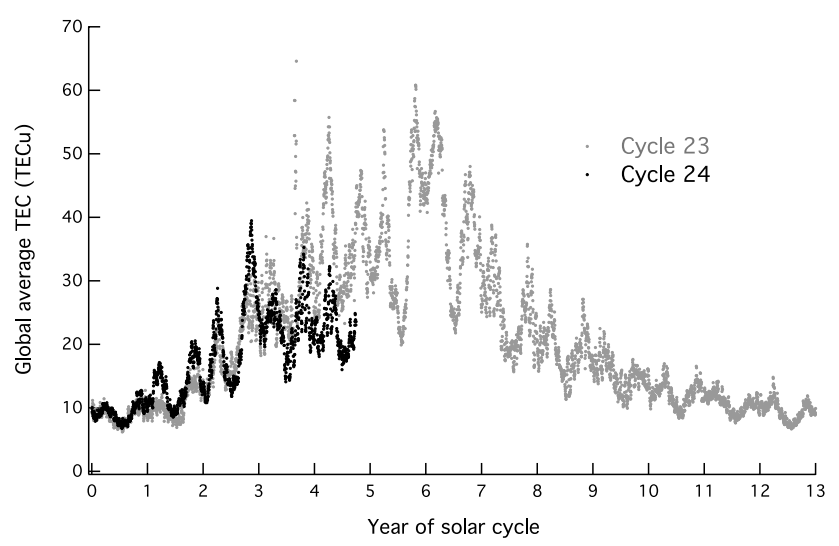

Figure 4. Daily global average TEC (TEC) of solar cycle 23 (grey points) and 24 (black points). The number labels on the $x$ axis denote the year counted from the beginning of each solar cycle.

in Fig. 5. In the years 2010-2011 the TEC value of SC24 is above the $+1 \sigma$ line of $\mathrm{SC} 23$ in particular at equinoctial peaks; after 2012 the TEC turned out to be along and later below the $-1 \sigma$ line, and this has been especially obvious since 2013.

\section{Discussion}

\subsection{Ionosphere in solar cycle 24}

The ionization of the neutral atmosphere to form the ionosphere is driven directly by solar irradiance. When the Sun is inactive in EUV emissions, e.g., during the very deep solar minimum of 2008-2009, the ionosphere is weakly produced (e.g., Liu et al., 2011a, b). Similarly, is the negative deviation of $f o \mathrm{~F} 2$ and $\overline{\mathrm{TEC}}$ a result of the slow rising and succedent low-level solar activity of SC24? Figures 6 and 7 present the SSN record (SIDC-team, 1954-2013) since SC19 and the solar F10.7 index since SC20, respectively, from which one can notice that

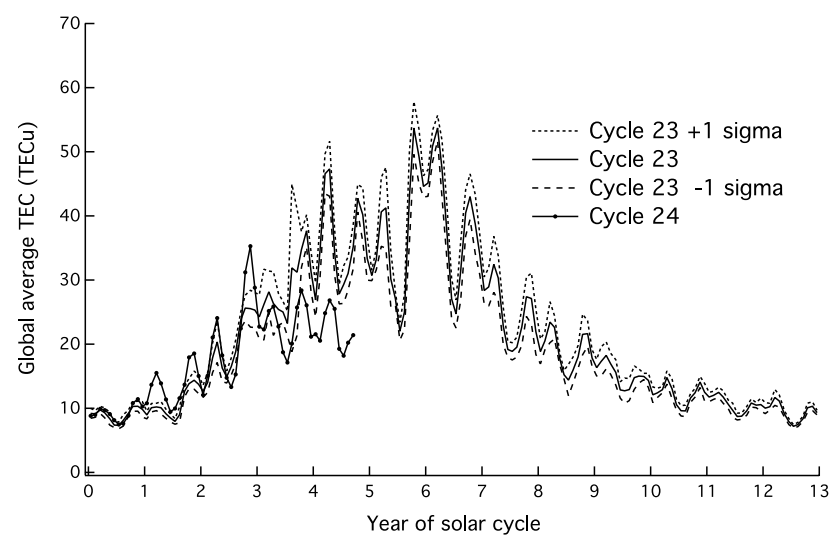

Figure 5. Similar to Fig. 4, but displayed are the monthly mean $\overline{\mathrm{TEC}}$ of both SC23 and SC24. The standard deviations $( \pm 1 \sigma)$ about the monthly mean $\overline{\text { TEC }}$ of SC23 are shown in dotted and dashed lines as well.

- The SSN stayed low after 2008-2009, and climbed slowly after the initiation of SC24. The low level of solar ionization could therefore essentially have produced the lower $f o \mathrm{~F} 2$ value relative to $\mathrm{SC} 19-23$. As shown in Fig. 6, the rising trend of 13-month smoothed SSN ended in 2012 (red solid line), which was directly reflected in $f o \mathrm{~F} 2$ and $\overline{\mathrm{TEC}}$, with significant negative deviations compared to previous cycles.

- With regard to precedent solar cycles, SC23 is also a relatively weak cycle, especially when the ascending phases of all cycles are compared. Before 2011, the slow rising of both SSN and the F10.7 index of SC24 seemed similar to SC23; the $\overline{\mathrm{TEC}}$ value of $\mathrm{SC} 24$ even exceeded the level of SC23, particularly in 2011 when the Sun burst around the equinoxes. However, the Sun calmed down afterward and the TEC of SC24 went significantly below the level of SC23.

Up to the latest SSN data from September 2013, the maximum monthly SSN of SC24 was 96.7 in November 2011 (SIDC-team, 1954-2013). This was found to be coincident with the peaks of $f o \mathrm{~F} 2$ and $\overline{\mathrm{TEC}}$ occurring around the fall equinox of 2011. In the ensuing two years, the solar activity went completely into a valley. Actually, SC24 has appeared as an unusually weak cycle with very late start and slow ascending phases compared to precedent cycles (e.g., Li et al., 2011). Our analysis of both single-station $f o \mathrm{~F} 2$ and global $\overline{T E C}$ data has revealed the effect of an inactive Sun on the ionosphere. In general, it is concluded that the ionosphere as a whole in SC24 has and will contain far fewer electrons, which noticeably deviates from other cycles during the space age. 


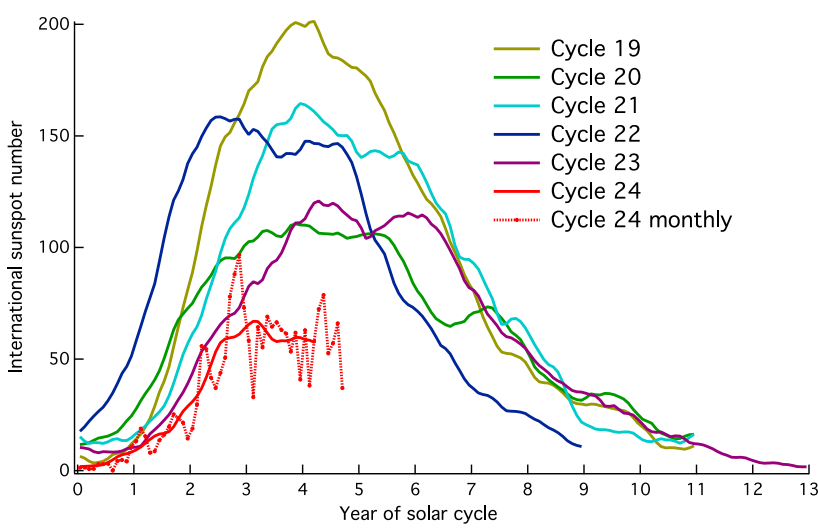

Figure 6. Sunspot number records since the beginning of SC19. The 13-month smoothed monthly SSN for each cycle is shown (solid lines), and the monthly SSN of SC24 till September 2013 is displayed (dotted line with symbols) as well.

\subsection{Contribution of solar irradiance}

As mentioned in Sect. 1, it was predicted that $\mathrm{SC} 24$ would reach its peak SSN of 90 in May 2013 (http:// www.swpc.noaa.gov/SolarCycle/SC24/index.html), whereas an observed SSN peak appeared in November 2011. Does it mean the Sun reached its maximum in 2011, revealing the peak level of the global ionosphere? Regarding the peak of SC24, two possible scenarios can be supposed. First, the peak occurred in November 2011, and the Sun has been in the descending phase since then. Secondly, SC24 will exhibit a double-peaked structure and the second maximum will be coming in two years. The latter scenario has a higher probability according to the latest solar observations.

Both of the last two cycles of SC22 and SC23 had twin peaks, and in either cycle SSN maximized in the first peak (see Fig. 6). However, in both SC22 and SC23 the second peak of the F10.7 index was equal to or exceeded the first one (see Fig. 7). The solar F10.7 index rather than SSN is an appropriate proxy for solar EUV emissions, which essentially determine the ionization of the atmosphere. This explains why in SC23 the second peak of $\overline{\mathrm{TEC}}$ is higher than the first one during the period of the years 2000-2002 (Fig. 4). Accordingly, although it is very likely that $\mathrm{SC} 24$ has passed its peak in terms of SSN, the maximum level of solar EUV emissions and the ionosphere is yet to come.

Figure 8 reveals the absolute and relative changes in $\overline{\mathrm{TEC}}$ and the F10.7 index of SC24 compared to those of SC23. Variations in the two parameters were not correlated in the first two years (2009-2010), when the Sun was quiet and the effects of non-solar factors were possibly important to the ionosphere, whereas after 2012, the negative deviations of $\overline{T E C}$ and the F10.7 index varied fairly synchronously, both of which were enlarged to the level of $-40 \%$ in 2013. This finding implies that the decline in solar irradiance caused a proportional decrease in global total ionospheric electron density, and quantitatively confirms the key role EUV emissions

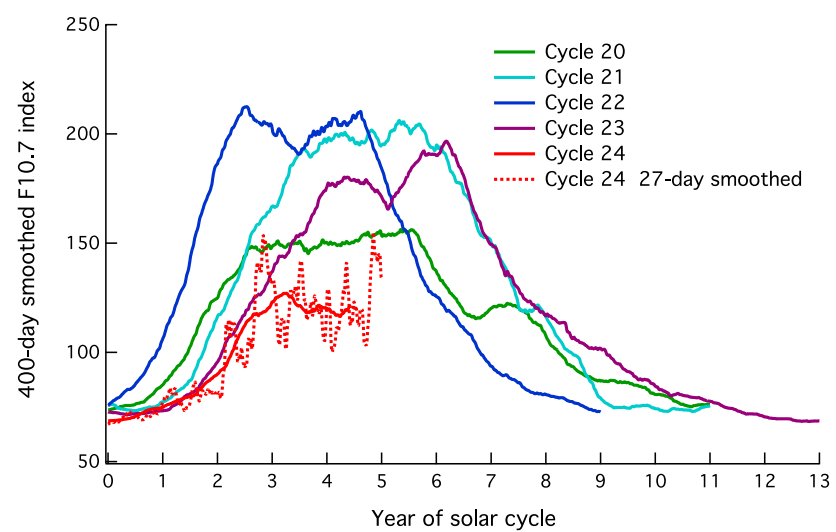

Figure 7. Solar F10.7 index since the beginning of SC20. The 400day (approximately 13-month) smoothed F10.7 index for each cycle is shown (solid lines), and for SC24 the 27-day smoothed F10.7 index till December 2013 is displayed (dotted line) as well.

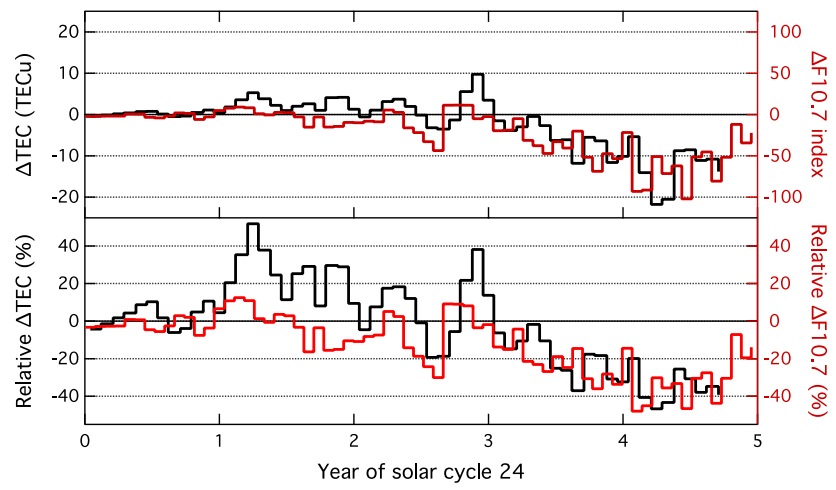

Figure 8. Absolute (upper) and relative (lower) changes in monthly mean $\overline{\text { TEC }}$ (left axis) and the 27-day mean F10.7 index (right axis) between SC24 and SC23. The number labels on the $x$ axis denote the year counted from the beginning of SC23/24.

played in controlling the ionosphere when the Sun was active. It thus further supports the prediction that the ionosphere of SC24 may have a second peak higher, if the ionization by EUV is stronger at that time. Despite the possibility of having a second peak, the Sun is much less active in SC24, resulting in significantly weaker ionization of the atmosphere, which may imply a continuing anomaly of the ionosphere around the solar maximum and in the remaining period of this solar cycle.

\subsection{Vision for solar cycle 24 and beyond}

Taking a long-term perspective, the last three solar cycles (1976-2008) have had greater solar activity than usual. Accordingly, this thirty-year period has been called a "grand solar maximum" (Lockwood et al., 2009). Some long-term predictions of solar activity further suggested that SC24, similar to other weak cycles in history, may begin a grand 
minimum. A period of quiet solar activity is thus expected, which may last until 2030 (e.g., Clilverd et al., 2006; Solanki and Krivova, 2011).

Given that SC24 is likely to be an unprecedented weak cycle in the space age, previous studies on the solar-terrestrial relations with an inactive Sun is of great value for better understanding of the characteristics, trajectories and consequences of SC24. These studies point to certain aspects of research that deserve close attention, such as a minimum in geomagnetic activity related to low solar and interplanetary magnetic fields and the disappearance of low-latitude coronal holes (Tsurutani et al., 2011), response of the ionosphere to extremely low solar EUV irradiance (Liu et al., 2011a, b), as well as the considerable energy input of high-speed solar wind streams (HSSs) and its impact on the thermosphereionosphere system (Lei et al., 2008a, b; Verkhoglyadova et al., 2013).

If the prediction of a grand minimum comes true, the terrestrial thermosphere and ionosphere will undertake a longterm change and appear systematically different from the cycles in the past half century. Taking into consideration the complicity in the thermospheric and ionospheric responses to various energy inputs, current modeling of the ionosphere may thus require revision to capture the contribution of an inactive Sun as well as the widely concerned impact of greenhouse gases and the long-term trend of evolution of the neutral and ionized upper atmosphere (e.g., Laštovička, 2012, 2013; Zhang et al., 2011; Zhang and Holt, 2013). An ultra weak solar cycle or grand solar minimum will also provide a suitable moment for researchers to continue with the investigation of non-solar forcing made during the declining phase and minimum of SC23, which have greatly improved our understanding of energy coupling between the lower and upper atmospheres (see the review articles by Laštovička, 2009 and Pancheva and Mukhtarov, 2012). Various sources of energy from below have been found effectively to disturb the ionosphere, such as the modulation of the ionosphere by planetary waves (Hao and Zhang, 2012), spread F triggered by gravity waves from cyclones in the troposphere (Xiao et al., 2009; Xiao et al., 2012), and ionospheric disturbances caused by upward propagation of atmospheric infrasonic waves (Hao et al., 2012, 2013).

From a space weather perspective, the behavior of the ionospheric plasma and its subsequent effect can be very different under quiet conditions, according to our experience during the solar minimum of 2008-2009. For example, analysis of data from the Defense Meteorological Satellite Program (DMSP) satellite shows that the occurrence probability of equatorial plasma bubbles (EPB) decreased from about $10 \%$ in the previous minimum to less than $5 \%$ in the minimum between SC23 and SC24 (Gentile et al., 2011); the $\boldsymbol{E} \times \boldsymbol{B}$ drift measured by the Communications/Navigation Outage Forecasting System (C/NOFS) satellite during this period is found to diverge from the expected climatology based on previous solar minima (Klenzing et al., 2013). Also, a weak solar cycle is not necessarily free of exceptional solar events. Solar cycle 14, for instance, had a very low peak SSN of 64, but during which two super magnetic storms occurred (Svalgaard et al., 2005). It should also be noted that the distribution of great storms differs in weak and strong solar cycles (Le et al., 2012). How a weakly ionized atmosphere responds to a super storm then provides an interesting question regarding the solar-terrestrial relationship.

\section{Conclusions}

Both the ionosonde $f o \mathrm{~F} 2$ data of Kokubunji in East Asia and global average TEC display a common feature of the global ionosphere, that since entering solar cycle 24 the ionosphere has in general been weakly produced. The $f o \mathrm{~F} 2$ values were significantly lower than the average level of SC1923 from the very beginning of SC24 on. The TEC, different from $f o \mathrm{~F} 2$, was equal to or even higher compared to $\mathrm{SC} 23$ in 2009-2011; however, since 2012, although the Sun became active, both the $f o \mathrm{~F} 2$ and $\overline{\mathrm{TEC}}$ data showed a negative departure from the average level of the recent historical cycles. The $f o \mathrm{~F} 2$ was at most $40 \%$ lower than SC19-23, or $5 \mathrm{MHz}$ in value; the deviation of $\overline{\mathrm{TEC}}$ could be up to $-50 \%$ relative to SC23, or $30 \mathrm{TECu}$ in value. In 2013, when the Sun was expected to approach its maximum, both the $f o \mathrm{~F} 2$ and $\overline{T E C}$ were more negatively deviated, indicating that the global ionosphere was far away from a normal solar maximum level. The synchronous negative deviation of $\overline{\mathrm{TEC}}$ and the F10.7 index during the years 2012-2013 indicates the control effect of solar EUV, which is responsible for the weak ionization of the global ionosphere. In summary, the ionosphere of SC24 has shown an ultra weak ionization to date, which can be attributed to the sluggish rising of the new cycle. Due to the insufficient data and knowledge on weak solar cycles, it is not easy to predict how the thermosphereionosphere system will react around the maximum period of SC24. What will happen through the remainder of SC24 still remains to be seen.

Acknowledgements. This research draws upon data provided by the WDC for Ionosphere and Space Weather, Tokyo, National Institute of Information and Communications Technology. Global TEC data were obtained from CODE at AIUB. The F10.7 index was obtained from the GSFC/SPDF OMNIWeb interface at http://omniweb.gsfc.nasa.gov. This work was supported by the National Basic Research Program of China (grant nos. 2012CB957801 and 2011CB811405), the National Natural Science Foundation of China (grant nos. 41274155 and 40904036), the Public Science and Technology Research Funds Projects of Ocean, the State Oceanic Administration of China (grant no. 201005017), and the Specialized Research Fund for State Key Laboratories.

Topical Editor S. Milan thanks J. Laštovička and one anonymous referee for their help in evaluating this paper. 


\section{References}

Araujo-Pradere, E. A., Redmon, R., Fedrizzi, M., Viereck, R., and Fuller-Rowell, T. J.: Some Characteristics of the Ionospheric Behavior During the Solar Cycle 23-24 Minimum, Sol. Phys., 274, 439-456, doi:10.1007/s11207-011-9728-3, 2011.

Clilverd, M. A., Clarke, E., Ulich, T., Rishbeth, H., and Jarvis, M. J.: Predicting Solar Cycle 24 and beyond, Space Weather, 4, S09005, doi:10.1029/2005SW000207, 2006

Emmert, J. T., Lean, J. L., and Picone, J. M.: Record-low thermospheric density during the 2008 solar minimum, Geophys. Res. Lett., 37, L12102, doi:10.1029/2010GL043671, 2010.

Gentile, L. C., Burke, W. J., Roddy, P. A., Retterer, J. M., and Tsunoda, R. T.: Climatology of plasma density depletions observed by DMSP in the dawn sector, J. Geophys. Res., 116, A03321, doi:10.1029/2010JA016176, 2011.

Hao, Y. and Zhang, D.: Ionospheric absorption and planetary wave activity in East Asia sector, Sci. China Tech. Sci., 55, 1264-1272, doi:10.1007/s11431-012-4799-4, 2012.

Hao, Y. Q., Xiao, Z., and Zhang, D. H.: Multi-instrument observation on co-seismic ionospheric effects after great Tohoku earthquake, J. Geophys. Res., 117, A02305, doi:10.1029/2011JA017036, 2012.

Hao, Y. Q., Xiao, Z., and Zhang, D. H.: Teleseismic magnetic effects (TMDs) of 2011 Tohoku earthquake, J. Geophys. Res., 118, 3914-3923, doi:10.1002/jgra.50326, 2013.

Hocke, K.: Oscillations of global mean TEC, J. Geophys. Res., 113, A04302, doi:10.1029/2007JA012798, 2008.

Kane, R. P.: Size of the coming solar cycle 24 based on Ohl's Precursor Method, final estimate, Ann. Geophys., 28, 1463-1466, doi:10.5194/angeo-28-1463-2010, 2010.

Kane, R. P.: An Estimate for the Size of Sunspot Cycle 24, Sol. Phys., 282, 87-90, doi:10.1007/s11207-012-0131-5, 2013.

Klenzing, J., Burrell, A. G., Heelis, R. A., Huba, J. D., Pfaff, R., and Simões, F.: Exploring the role of ionospheric drivers during the extreme solar minimum of 2008, Ann. Geophys., 31, 2147-2156, doi:10.5194/angeo-31-2147-2013, 2013.

Laštovička, J.: Lower ionosphere response to external forcing: A brief review, Adv. Space Res., 43, 1-14, doi:10.1016/j.asr.2008.10.001, 2009.

Laštovička, J.: On the role of ozone in long-term trends in the upper atmosphere-ionosphere system, Ann. Geophys., 30, 811816, doi:10.5194/angeo-30-811-2012, 2012.

Laštovička, J.: Trends in the upper atmosphere and ionosphere: Recent progress, J. Geophys. Res., 118, 3924-3935, doi:10.1002/jgra.50341, 2013.

Le, G., Cai, Z., Wang, H., and Zhu, Y.: Solar cycle distribution of great geomagnetic storms, Astrophys. Space. Sci., 339, 151-156, doi:10.1007/s10509-011-0960-y, 2012.

Lei, J., Thayer, J. P., Forbes, J. M., Sutton, E. K., Nerem, R. S., Temmer, M., and Veronig, A. M.: Global thermospheric density variations caused by high-speed solar wind streams during the declining phase of solar cycle 23, J. Geophys. Res., 113, A11303, doi:10.1029/2008JA013433, 2008a.

Lei, J., Thayer, J. P., Forbes, J. M., Wu, Q., She, C., Wan, W., and Wang, W.: Ionosphere response to solar wind high-speed streams, Geophys. Res. Lett., 35, L19105, doi:10.1029/2008GL035208, 2008b.

Li, K. J., Feng, W., Liang, H. F., Zhan, L. S., and Gao, P. X.: A brief review on the presentation of cycle 24 , the first integrated solar cycle in the new millennium, Ann. Geophys., 29, 341-348, doi:10.5194/angeo-29-341-2011, 2011.

Liu, L., Wan, W., Ning, B., and Zhang, M.: Climatology of the mean total electron content derived from GPS global ionospheric maps, J. Geophys. Res., 114, A06308, doi:10.1029/2009JA014244, 2009.

Liu, L., Chen, Y., Le, H., Kurkin, V. I., Polekh, N. M., and Lee, C.-C.: The ionosphere under extremely prolonged low solar activity, J. Geophys. Res. Space, 116, A04320, doi:10.1029/2010JA016296, 2011a.

Liu, L., Wan, W., Chen, Y., and Le, H.: Solar activity effects of the ionosphere: A brief review, Chinese Sci. Bull., 56, 1202-1211, doi:10.1007/s11434-010-4226-9, 2011b.

Lockwood, M.: Was UV spectral solar irradiance lower during the recent low sunspot minimum?, J. Geophys. Res., 116, D16103, doi:10.1029/2010JD014746, 2011.

Lockwood, M., Rouillard, A. P., and Finch, I. D.: The Rise and Fall of Open Solar Flux During the Current Grand Solar Maximum, Astrophys. J., 700, 937-944, doi:10.1088/0004-637X/700/2/937, 2009.

Mannucci, A. J., Wilson, B. D., Yuan, D. N., Ho, C. H., Lindqwister, U. J., and Runge, T. F.: A global mapping technique for GPSderived ionospheric total electron content measurements, Radio Sci., 33, 565-582, doi:10.1029/97RS02707, 1998.

Owens, M. J., Lockwood, M., Barnard, L., and Davis, C. J.: Solar cycle 24: Implications for energetic particles and longterm space climate change, Geophys. Res. Lett., 38, L19106, doi:10.1029/2011GL049328, 2011.

Pancheva, D. and Mukhtarov, P.: Global Response of the Ionosphere to Atmospheric Tides Forced from Below: Recent Progress Based on Satellite Measurements, Space Sci. Rev., 168, 175-209, doi:10.1007/s11214-011-9837-1, 2012.

Pesnell, W. D.: Predictions of Solar Cycle 24, Sol. Phys., 252, 209 220, doi:10.1007/s11207-008-9252-2, 2008.

Pesnell, W. D.: Solar Cycle Predictions (Invited Review), Sol. Phys., 281, 507-532, doi:10.1007/s11207-012-9997-5, 2012.

Russell, C. T., Luhmann, J. G., and Jian, L. K.: How unprecedented a solar minimum?, Rev. Geophys., 48, RG2004, doi:10.1029/2009RG000316, 2010.

Schaer, S., Gurtner, W., and Feltens, J.: IONEX: The ionosphere map exchange format version 1, in: Proceedings of the IGS AC Workshop, Darmstadt, Germany, Vol. 9, 1998.

SIDC-team: The International Sunspot Number, Monthly Report on the International Sunspot Number, online catalogue, available at: http://www.sidc.be/sunspot-data/ (last access: December 2013), 1954-2013.

Solanki, S. K. and Krivova, N. A.: Analyzing Solar Cycles, Science, 334, 916-917, doi:10.1126/science.1212555, 2011.

Solomon, S. C., Woods, T. N., Didkovsky, L. V., Emmert, J. T., and Qian, L.: Anomalously low solar extreme-ultraviolet irradiance and thermospheric density during solar minimum, Geophys. Res. Lett., 37, L16103, doi:10.1029/2010GL044468, 2010.

Svalgaard, L., Cliver, E. W., and Kamide, Y.: Sunspot cycle 24: Smallest cycle in 100 years?, Geophys. Res. Lett., 32, L01104, doi:10.1029/2004GL021664, 2005.

Tsurutani, B. T., Echer, E., and Gonzalez, W. D.: The solar and interplanetary causes of the recent minimum in geomagnetic activity (MGA23): a combination of midlatitude small coronal holes, low IMF BZ variances, low solar wind speeds and low solar mag- 
netic fields, Ann. Geophys., 29, 839-849, doi:10.5194/angeo-29839-2011, 2011.

Verkhoglyadova, O. P., Tsurutani, B. T., Mannucci, A. J., Mlynczak, M. G., Hunt, L. A., and Runge, T.: Variability of ionospheric TEC during solar and geomagnetic minima (2008 and 2009): external high speed stream drivers, Ann. Geophys., 31, 263-276, doi:10.5194/angeo-31-263-2013, 2013.

Xiao, S.-G., Xiao, Z., Shi, J.-K., Zhang, D.-H., Feng, X.-S., Hao, Y.-Q., and Huang, W.-Q.: Observational facts in revealing a close relation between acoustic-gravity waves and midlatitude spread F, J. Geophys. Res., 114, A01303, doi:10.1029/2008JA013747, 2009.
Xiao, S., Shi, J., Zhang, D., Hao, Y., and Huang, W.: Observational study of daytime ionospheric irregularities associated with typhoon, Sci. China Tech. Sci., 55, 1302-1304, doi:10.1007/s11431-012-4816-7, 2012.

Zhang, S.-R. and Holt, J. M.: Long-term ionospheric cooling: Dependency on local time, season, solar activity, and geomagnetic activity, J. Geophys. Res., 118, 3719-3730, doi:10.1002/jgra.50306, 2013.

Zhang, S.-R., Holt, J. M., and Kurdzo, J.: Millstone Hill ISR observations of upper atmospheric long-term changes: Height dependency, J. Geophys. Res., 116, A00H05, doi:10.1029/2010JA016414, 2011. 